\title{
Multihadron decays of new mesons
}

\author{
K. Koller and T. F. Walsh \\ Deutsches Elektronen-Synchrotron DESY, Hamburg, Germany \\ (Received 15 December 1975)
}

\begin{abstract}
We discuss the hadronic decays of the new $I=0$ mesons seen in $e^{+} e^{-}: J(\psi)$ or $\psi^{\prime}$ with $G=-$ and $P_{c}(\chi)$ or $X$ with $G=+$. We present some isospin inequalities for $I=0$ pure pionic final states, and a discussion of $K \bar{K}$ and $\eta, \eta^{\prime}$ fractions. We also present a statistical-model analysis of pion final states, and conclude that a large fraction of hadronic $J(\psi)$ decays contain something besides pions and $K \bar{K}$-probably $\eta$ and $\eta^{\prime}$, possibly radiative modes.
\end{abstract}

\section{INTRODUCTION}

There is now plenty of evidence that the new resonances $J(\psi)(3.1)^{1,2}$ and $\psi^{\prime}(3.7)^{3}$ are hadrons, with $J^{P C}=1^{-}$, odd $G$ parity, and isospin zero. Isospin-violating decays take place via $J \rightarrow 1 \gamma \rightarrow$ hadrons. Recently, even- $C$ states have been found at 3.4 and $3.5 \mathrm{GeV}$, reached via $\psi^{\prime} \rightarrow P_{c}(\chi)$ $+\gamma \cdot{ }_{0}^{4,5,6}$ There is also some evidence for a state at $2.8 \mathrm{GeV}, J \rightarrow X(2.8)+\gamma, X \rightarrow \gamma \gamma$ and $p \bar{p} .^{6,7}$ The former states have the decay modes (3.4), $(3.5)-2\left(\pi^{+} \pi^{-}\right), \rightarrow 3\left(\pi^{+} \pi^{-}\right) .{ }^{5}$ This shows that they have even $G$ and even $I$. Evidence that $(3.4) \rightarrow \pi^{+} \pi^{-}$and $K \bar{K}$ indicates $I=0$ (see Ref. 5 ); so would a $p \bar{p}$ final state.

In view of the present experimental situation, it is useful to study the hadronic decays of $J, \psi^{\prime}$, and $\chi$, using the fact that they have $I=0$, or assuming it. That is what we will do here. First, we present rigorous isospin bounds according to the methods of Llewellyn Smith and Pais, ${ }^{8}$ also extending the old isospin statistical model of Pais ${ }^{9}$ to a larger number of pions. To this we add a few remarks on final states with $K \bar{K}, \eta$, and $\eta^{\prime}$. The rest of the paper contains a discussion of the present experimental data.

We hope that this material will be useful to experimentalists and offer a partial view of the final hadron states in the decay of the new particles seen in $e^{+} e^{-}$. This may be of importance in view of the very small hadronic width of these mesons. Perhaps a study of final states will help us understand the mechansim which suppresses the hadronic width.

\section{PIONS, KAONS, $\eta$, and $\eta^{\prime}$}

\section{A. Pions}

In this sub section we present isospin bounds and a statistical model for pure $I=0$ pionic final states. The method goes back to a very useful paper of Pais. ${ }^{9}$ We review his method briefly. For such bounds to be useful in practice, final state pions must not arise from $\eta$ or $\eta^{\prime}$ decay. We will discuss this at the end of the next subsection. We will assume here that events containing $K \bar{K}, \eta, \eta^{\prime}$ and nucleon-antinucleon pairs can be segregated out and treated separately.

Isospin- 0 states with $N$ pions can be labelled by three numbers $N_{1}, N_{2}, N_{3}$, where $N_{1} \geq N_{2} \geq N_{3} \geq 0$, $N_{1}-N_{3}$ even, $N_{2}-N_{3}$ even, $N=N_{1}+N_{2}+N_{3} .{ }^{10}$ These numbers designate a Young tableau of the unitary group in three dimensions $\mathrm{U}(3)$, and constitute a labeling of all unitary representations of this group. All the consequences of isospin conservation for $I=0 \mathrm{de}$ cay to pions then follow from the fact that the isospin group $\mathrm{SU}(2)_{I}$ is a subgroup of this $\mathrm{U}(3)$. Labeling a charge partition of $m_{c}\left(\pi^{+} \pi^{-}\right)$pairs and $m_{0} \pi^{0}$ mesons by $\left(m_{c}, m_{0}\right)$, the probability that this will be found in a state $\left(N_{1} N_{2} N_{3}\right)$ is given by a Pais coefficient ${ }^{9}\left[N_{1} N_{2} N_{3} \mid m_{c} m_{0}\right]$, normalized so that $\sum_{m_{c} m_{0}}\left[N_{1} N_{2} N_{3} \mid m_{c} m_{0}\right]=1$ for fixed $N=2 m_{c}+m_{0}$. These coefficients can be calculated by combinatoric methods and by establishing nontrivial identities between them. The branching ratio $\Gamma$ for a state containing only $N$ pions is

$\Gamma\left(2 m_{c} \pi^{c}, m_{0} \pi^{0}\right)=\frac{1}{\Delta} \sum_{N_{i}} \alpha\left(N_{1} N_{2} N_{3}\right)\left[N_{1} N_{2} N_{3} \mid m_{c} m_{0}\right]$,

where

$\alpha\left(N_{1} N_{2} N_{3}\right)=\rho\left(N_{1} N_{2} N_{3}\right) K\left(N_{1} N_{2} N_{3}\right)$,

$\rho\left(N_{1} N_{2} N_{3}\right)=\frac{N !\left(N_{1}-N_{2}+1\right)\left(N_{1}-N_{3}+2\right)\left(N_{2}-N_{3}+1\right)}{\left(N_{1}+2\right) !\left(N_{2}+1\right) !\left(N_{3}\right) !}$,

and the non-negative $K\left(N_{1} N_{2} N_{3}\right)$ take care of the (unknown) dynamics; $\rho\left(N_{1} N_{2} N_{3}\right)$ is the dimensionality of the $\left(N_{1} N_{2} N_{3}\right)$ representation of $S_{3}$, and simply counts the number of available states (measures the isospin phase space). The normalizations are 


$$
\begin{aligned}
& \Delta=\sum_{N_{i}} \alpha\left(N_{1} N_{2} N_{3}\right), \\
& \sum_{m_{c}, m_{0}} \Gamma\left(2 m_{c} \pi^{c}, m_{0} \pi^{0}\right)=1,
\end{aligned}
$$

where $N=2 m_{c}+m_{0}$ and the sum $N_{i}$ is over all $I=0$ partitions of fixed $N=N_{1}+N_{2}+N_{3}$. Because of the linearity of (1), the problem of finding bounds for
$\Gamma$ reduces to that of finding that $\left[N_{1} N_{2} N_{3} \mid m_{c} m_{0}\right]$ which is largest or smallest for a given $N$. The bound corresponds to $K\left(N_{1} N_{2} N_{3}\right)=1$ for that partition and to zero for all others. The bounds for $N$ odd can be applied to $J, \psi^{\prime} \rightarrow\left(N-m_{0}\right) \pi^{c} m_{0} \pi^{0}, m_{0}$ $=1,3 \ldots$, or to single-photon $e^{+} e^{-}$annihilation to pions; the bounds for $N$ even can be applied to $\chi_{i}\left(N-m_{0}\right) \pi^{c} m_{0} \pi^{0}, m_{0}=0,2 \ldots$, and to $e^{+} e^{-}$. The bounds for $N$ odd are

$$
\left.\begin{array}{l}
\left.\begin{array}{ll}
N=3: & 1 \\
N=5: & \frac{2}{3} \\
N=7: & \frac{1}{3} \\
N \geq 9: & 0
\end{array}\right\} \leq \Gamma\left((N-1) \pi^{c}, 1 \pi^{0}\right) \leqslant \frac{2^{N-3}\left[\left(\frac{N-3}{3}\right) !\right]^{2}}{(N-2) !}, N \geqslant 3 \\
5 \leq N \leq 13: \frac{2^{N-4}\left[\left(\frac{N-3}{2}\right) !\right]^{2}}{(N-2) !} \\
N \geq 15:
\end{array}\right\} \leq \Gamma\left((N-3) \pi^{c}, 3 \pi^{0}\right) \leqslant \begin{cases}\frac{1}{3}, & N=5 \\
\frac{2}{3}, & N=7 \\
\frac{2^{N-9}\left[\left(\frac{N-9}{2}\right) !\right]^{2}}{(N-8) !}, & N \geq 9 .\end{cases}
$$

The bounds for $N$ even are

$$
\begin{aligned}
& \left.\begin{array}{ll}
N=2: & \frac{2}{3} \\
N=4: & \frac{1}{3} \\
N \geqslant 6: & 0
\end{array}\right\} \leqslant \Gamma\left(N \pi^{c}\right) \leqslant \frac{2^{N}\left[\left(\frac{N}{2}\right) !\right]^{2}}{(N+1) !}, N \geqslant 2, \\
& \left.\begin{array}{ll}
2 \leqslant N \leqslant 10: & \frac{2^{N-1}\left[\left(\frac{N}{2}\right) !\right]^{2}}{(N+1) !} \\
N \geqslant 12: & 0
\end{array}\right\} \leqslant \Gamma\left((N-2) \pi^{c}, 2 \pi^{0}\right) \leqslant \begin{cases}\frac{1}{3}, & N=2, \\
\frac{2}{3}, & N=4, \\
\frac{2^{N-6}\left[\left(\frac{N-6}{2}\right) !\right]^{2}}{(N-5) !}, & N \geqslant 6 .\end{cases}
\end{aligned}
$$

The existence of nonzero lower bounds for low $N$ may be especially useful. ${ }^{11}$ Note that in the limit $N-3 m_{0} \rightarrow \infty$ the branching ratios fulfill

$$
0 \leqslant \Gamma\left(\left(N-m_{0}\right) \pi^{c}, m_{0} \pi^{0}\right) \leqslant\left[\frac{\pi}{2}\left(N-3 m_{0}\right)\right]^{1 / 2} .
$$

We mention that the isospin bounds arise when all but one certain $K\left(N_{1} N_{2} N_{3}\right)$ are zero. The opposite extreme is to assume that they are all equal. This produces an isospin statistical model for fixed $N$, with each $\left[N_{1} N_{2} N_{3} \mid m_{c} m_{0}\right]$ weighted by the number of states $\rho\left(N_{1} N_{2} N_{3}\right)$ corresponding to this class. ${ }^{9}$ Dynamical effects will give deviations from this model. Being unable to find a general formula, we extended the $\left[N_{1} N_{2} N_{3} \mid m_{c} m_{0}\right]$ tables of Pais $(N \leqslant 8)$ up to $N=13$ for odd $N$ and to $N=10$ for even $N$ 。 The results (including $N \leqslant 8$ for completeness) can be found in Tables I(a) and I(b).

It is less obvious how to construct a statistical model for the pion distribution in $N$. The simplest possibility, which we will use, is to assume a Poisson distribution,

$$
P(N)=k^{-1} \frac{\langle N\rangle^{N}}{N !} e^{-\langle N\rangle}
$$

for $N$ odd or even, $2 \leqslant N \leqslant 22$, normalizing by $\sum P(N)=1$ with the sum over even or odd $N$ as the case requires. We will use this to estimate the total pion branching ratio. 


\section{B. Kaons, $\eta$, and $\eta^{\prime}$}

The situation for final states with $K \bar{K}$ is more obscure. We can obtain a few simple isospin relations for such final states by exploiting the fact that the initial state has $I=0.0^{12}$ There are then two amplitudes for inclusively produced $K \bar{K}$ with $I=0$ and $I=1$. Eliminating the $I=0,1$ interference term we find the inclusive rate $d \sigma(a b X)$

$$
d \sigma\left(K^{0} \bar{K}^{0} X^{0}\right)+d \sigma\left(K^{+} K^{-} X^{0}\right) \geqslant d \sigma\left(K^{+} \bar{K}^{0} X^{-}\right),
$$

and bounding the interference term,

$$
\left[d \sigma\left(K^{0} \bar{K}^{0} X^{0}\right)-d \sigma\left(K^{+} K^{-} X^{0}\right)\right]^{2} \leqslant 4 d \sigma\left(\mathrm{K}^{+} \bar{K}^{0} X^{-}\right)\left[d \sigma\left(K^{0} \bar{K}^{0} X^{0}\right)+d \sigma\left(K^{+} K^{-} X^{0}\right)-d \sigma\left(K^{+} \bar{K}^{0} X^{-}\right)\right] .
$$

These are summed over the internal variables of $X$ but not over $K \bar{K}$ momenta. For pure $I=1 K \bar{K}$, (7) is an equality and of course $d \sigma\left(K^{0} \bar{K}^{0} X^{0}\right)=d \sigma\left(K^{+} K^{-} X^{0}\right)$ for vanishing isoscalar/ isovector interference.

It is possible to do better for exclusive final states. For $\bar{K} K \pi$ (or nucleon-antinucleon-pion) we have pure $I=1$ and $^{13}$

$$
\begin{aligned}
2 \bar{K}^{0} K^{0} \pi^{0} & =2 K^{+} K^{-} \pi^{0} \\
& =K^{+} \bar{K}^{0} \pi^{-} .
\end{aligned}
$$

For $K \bar{K} \pi \pi$ we can get strong relations only after integrating over all $\pi \pi$ momenta. It is also possible to hold the $\pi \pi$ mass fixed (not integrated out), so long as the c.m. $\pi \pi$ angles are integrated over. The isoscalar/isovector interference then vanishes; this is because $I=0$ and $I=1$ correspond to different $\pi \pi$ angular momenta. Then

$$
\begin{aligned}
& K^{+} \bar{K}^{0} \pi^{-} \pi^{0}=K^{-} K^{0} \pi^{+} \pi^{0}, \\
& K^{+} K^{-} \pi^{+} \pi^{-}=\bar{K}^{0} K^{0} \pi^{+} \pi^{-}, \\
& K^{+} K^{-} \pi^{0} \pi^{0}=\bar{K}^{0} K^{0} \pi^{0} \pi^{0}, \\
& 2 K^{+} K^{-} \pi^{+} \pi^{-}=4 K^{+} K^{-} \pi^{0} \pi^{0}+K^{+} \bar{K}^{0} \pi^{-} \pi^{0} .
\end{aligned}
$$

This last relation enables us to get nearly unmeasurable modes like $K^{+} K^{-} \pi^{0} \pi^{0}$ and $K^{0} \overline{K^{0}} \pi^{0} \pi^{0}$ from measurable ones.

Note that if we again appeal to statistical considerations it appears reasonable to weight the isovector : isoscalar ratios by 3:1 and to ignore the interference of the two. Then

$$
\begin{aligned}
d \sigma\left(\bar{K}^{0} K^{0} X^{0}\right) & =d \sigma\left(K^{+} K^{-} X^{0}\right) \\
& =d \sigma\left(K^{+} \bar{K}^{0} X^{-}\right) \\
& =d \sigma\left(K^{-} K^{0} X^{+}\right) .
\end{aligned}
$$

Inclusive isospin relations can also be derived for other combinations of final-state particles using the zero isospin of the initial state. Just as an example, consider inclusive $K \pi$ states with isospin $\frac{1}{2}$ amplitude $a$ and $I=\frac{3}{2}$ amplitude $b$. Then

$$
\begin{aligned}
& d \sigma\left(K^{+} \pi^{-}\right)=\frac{1}{3}|a|^{2}+\frac{1}{12}|b|^{2}+\frac{1}{3} \operatorname{Re} a b^{*}, \\
& d \sigma\left(K^{+} \pi^{0}\right)=\frac{1}{6}|a|^{2}+\frac{1}{6}|b|^{2}+\frac{1}{3} \operatorname{Re} a b^{*}, \\
& d \sigma\left(K^{0} \pi^{+}\right)=\frac{1}{3}|a|^{2}+\frac{1}{12}|b|^{2}-\frac{1}{3} \operatorname{Re} a b^{*}, \\
& d \sigma\left(K^{0} \pi^{0}\right)=\frac{1}{6}|a|^{2}+\frac{1}{6}|b|^{2}-\frac{1}{3} \operatorname{Re} a b^{*}, \\
& d \sigma\left(K^{+} \pi^{+}\right)=d \sigma\left(K^{0} \pi^{-}\right)=\frac{1}{4}|b|^{2},
\end{aligned}
$$

from which we obtain the equalities

$$
\begin{aligned}
& d \sigma\left(K^{+} \pi^{-}\right)+d \sigma\left(K^{0} \pi^{0}\right)=d \sigma\left(K^{+} \pi^{0}\right)+d \sigma\left(K^{0} \pi^{+}\right), \\
& \begin{aligned}
2\left[d \sigma\left(K^{+} \pi^{0}\right)+d \sigma\left(K^{0} \pi^{0}\right)\right] \\
=
\end{aligned}
\end{aligned}
$$

Of course we can go back to (12) and derive inequalities for inclusive rates or for different charge partitions of exclusive channels. In deriving relations for exclusive channels [e.g. Eq. $\left.\left(10^{\prime}\right)\right]$, it is necessary to remember to include combinatorial factors for identical particles.

In order to obtain relations for final states with $\eta$ or $\eta^{\prime}$, we need to make assumptions beyond isospin invariance. For this reason we will make only a few brief comments, and these are model-dependent.

It is widely believed that the new mesons seen so far in $e^{+} e^{-}$and in hadron collisions are composites of new heavy quarks, and that these states are $\mathrm{SU}(3)$ singlets [ignoring $\mathrm{SU}(3)$ breaking]. If this is so, and if we can ignore $J \rightarrow 1 \gamma \rightarrow$ hadrons in first approximation, we expect all octet mesons to be produced with equal rate, and ${ }^{14}$

$$
\begin{aligned}
d \sigma\left(K^{+} X^{-}\right) & =d \sigma\left(K_{s}^{0} X^{0}\right) \\
& =d \sigma\left(\eta X^{0}\right)
\end{aligned}
$$

(pure octet $\eta$ ). If in addition $\eta^{\prime}$ is an SU(3) singlet built of the familiar $u, d, s$ quarks only,

$$
d \sigma(\eta X)=d \sigma\left(\eta^{\prime} X\right) \text {. }
$$

In applying this, remember that $\eta^{\prime} \rightarrow \eta \pi \pi$ is a prominent decay. Even if SU(3) breaking suppresses production of heavy pseudoscalars $K, \eta, \eta^{\prime}$ relative to pions by a large factor, (14) and (15) may still be good to 20-40\% typical for mass splitting 
corrections. However, it is unlikely that phase space alone will suppress single $\eta$ or $\eta^{\prime}$ production relative to $K \bar{K}$ 。

Under the same assumptionis as (13), plus an ideally mixed $\phi$, we have

$$
\frac{\Gamma\left(\eta^{\prime} \phi\right)}{\Gamma(\eta \phi)}=\frac{1}{2}
$$

Since $\eta^{\prime}$ can in principle have a small admixture of SU(3)-singlet heavy quarks, , (16) may be wrong by a significant factor. If this admixture is at the $\lessgtr 10 \%$ level we would expect only $\$ 20 \%$ effects on (16) and (15). It is of evident: importance to look for $\eta$ and $\eta^{\prime}$ in $J$ and $\psi^{\prime}$ decays. ${ }^{15}$

\section{EXPERINIENT}

Although new data on the decays of $J, \psi^{\prime}$, and $\chi$ might lead to changes in any detailed phenomenological analysis, we think it worthwhile to present at least a brief discussion of $J, \psi^{\prime}$, and $\chi$ decays. Experimentally, the decays $J \rightarrow 2 m_{c} \pi^{c} 1 \pi^{0}$ have been inferred for $m_{c}=1,2,3,4$ from the missing momentum and mass recoiling against $m_{c}\left(\pi^{*} \pi^{-}\right)$. Resonances (e.g. $\omega \pi^{*} \pi^{-}$and $\rho 3 \pi$ ) have been identified for $m_{c}=2 .{ }^{16}$ The experimentally measured branching ratios are $\sim \leqslant 1.9 \%$ for $m_{c}=1,4 \pm 1 \%$ for $m_{c}=2$, $2.9 \pm 0.7 \%$ for $m_{c}=3$, and $0 . \mathcal{c}_{ \pm} \pm 0.3 \%$ for $m_{c}=4 .^{16}$ We now assume that the $G=-$ states are reached through an isospin-conserving process and that, further, one may apply the isospin statistical model for fixed $N=7,9$ (the total $5 \pi$ rate is given by the isospin conservation alone). From Table I(a) we infer from the experimentally measured $\Gamma\left((N-1) \pi^{c}, 1 \pi^{0}\right)$ the following total $N$ pion branching ratios: $6 \%$ for $N=5,7 \%$ for $N=7$, and $3.7 \%$ for $N=9$. In order to obtain the total pion branching ratio of $J$, we correct for unmeasured decays with $N \geqslant 11$ using the Poisson distribution, Eq. (6), for $N$ odd, $3 \leqslant N \leqslant 22$. Fitting the numbers just quoted we find $\langle N\rangle=7$, and the entries in Table II (in percent). This gives us a total branching ratio $J \rightarrow$ (odd number of pions)

$$
\Gamma(J \rightarrow N \pi, \text { odd }) \approx 23 \% \text {. }
$$

The error in this number $i_{\text {is }}$ at least $\pm 5 \%$. Notice that for these decays $\left\langle N_{\mathrm{ch}}\right\rangle \approx 4.7$. Also, the as yet unobserved modes $J \rightarrow 10 \pi^{c} 1 \pi^{0}$ and $12 \pi^{c} 1 \pi^{0}$ are predicted to be very small: $0.3 \%$ and $0.05 \%$.

Because some of this may be useful for the $\chi$ states, we also present the same statistical-model expectation for $G=+$ pion decays of $\chi$, using $\langle N\rangle=7.5$ and normalizing the total to $100 \%$ (i.e. branching ratios normalized to the total of all pion isospin-conserving rates, not to the total widths). This is shown in Table III.

It is interesting (if somewhat risky) to attempt
TABLE I. Statistical-model branching ratios

\begin{tabular}{|c|c|c|c|c|c|c|}
\hline \multicolumn{7}{|c|}{$N$ odd } \\
\hline$m_{0}$ & 1 & 3 & 5 & 7 & 9 & 11 \\
\hline 3 & 1 & & & & & \\
\hline 5 & $\frac{2}{3}$ & $\frac{1}{3}$ & & & & \\
\hline 7 & $\frac{5}{12}$ & $\frac{6}{12}$ & $\frac{1}{12}$ & & & \\
\hline 9 & $\frac{14}{58}$ & $\frac{31}{58}$ & $\frac{12}{58}$ & $\frac{1}{58}$ & & \\
\hline 11 & $\frac{126}{951}$ & $\frac{448}{951}$ & $\frac{314}{951}$ & $\frac{60}{951}$ & $\frac{3}{951}$ & \\
\hline 13 & $\frac{396}{5649}$ & $\frac{2070}{5649}$ & $\frac{2300}{5649}$ & $\frac{790}{5649}$ & $\frac{90}{5649}$ & $\frac{3}{5649}$ \\
\hline \multicolumn{7}{|c|}{$N$ even } \\
\hline${ }_{N} \backslash^{m_{0}}$ & 0 & 2 & 4 & 6 & 8 & 10 \\
\hline 2 & $\frac{2}{3}$ & $\frac{1}{3}$ & & & & \\
\hline 4 & $\frac{6}{15}$ & $\frac{8}{15}$ & $\frac{1}{15}$ & & & \\
\hline 6 & $\frac{20}{105}$ & $\frac{66}{105}$ & $\frac{18}{105}$ & $\frac{1}{105}$ & & \\
\hline 8 & $\frac{70}{819}$ & $\frac{440}{819}$ & $\frac{276}{819}$ & $\frac{32}{819}$ & $\frac{1}{819}$ & \\
\hline 10 & $\frac{252}{6633}$ & $\frac{2590}{6633}$ & $\frac{2960}{6633}$ & $\frac{780}{6633}$ & $\frac{50}{6633}$ & $\frac{1}{6633}$ \\
\hline
\end{tabular}
$\Gamma\left(\left(N-m_{0}\right) \pi^{c}, m_{0} \pi^{0}\right)$.

to find the hadronic branching ratio $(86 \pm 2 \%)$ of the $J$ by adding together different classes of events. A possibly large contribution comes from hadronic events with $\geqslant 1 K \bar{K}$ pair (plus pions). To estimate this we can use a couple of simple observations. First, if not more than one $K \bar{K}$ pair is present and if we count events with $K_{s} K_{s}$ twice, then the fraction of hadronic events with $K \bar{K}$ is just equal to the fraction with a $K^{-}$plus the fraction with a $K_{S}$. This is all that has been measured directly so far. Going back to Sec. IIB, $K^{-}=K_{S}$ for a statistical distribution. The fraction of purely hadronic events in which $\mathrm{K}^{-}$has momentum $p \leqslant 0.7 \mathrm{GeV}$ at $J$ is $\approx 14 \% .^{17}$ Since corrections due to $K^{-}$with $p>0.7 \mathrm{GeV}$ and events with $2 K \bar{K}$ work in opposite

TABLE II. Statistical-model branching ratios (in percent) $\Gamma\left(\left(N-m_{0}\right) \pi^{c}, m_{0} \pi^{0}\right)$ for $G$-conserving decays of $J$.

\begin{tabular}{rlllllll}
\hline \hline$m_{0}$ & 1 & 3 & 5 & 7 & 9 & 11 & $\Gamma_{N}^{\text {total }}$ \\
\hline 3 & & & & & & & 2.4 \\
5 & 4.0 & 2.0 & & & & & 6 (input) \\
7 & 3.0 & 3.5 & 0.6 & & & & 7 \\
9 & 1.0 & 2.5 & 1.0 & 0.1 & & 4.6 \\
11 & 0.26 & 0.94 & 0.66 & 0.13 & 0.01 & 2 \\
13 & 0.05 & 0.26 & 0.29 & 0.10 & 0.01 & 0.0004 & 0.7 \\
& & & & & \multicolumn{3}{c}{$\sum_{N \geq 15} \Gamma_{N}^{\text {total }}=0.2$} \\
& & & & & & \\
\hline \hline
\end{tabular}


TABLE III. Statistical-model branching ratios (in percent) for $G=+, N$ even, $\Gamma\left(\left(N-m_{0}\right) \pi^{c}, m_{0} \pi^{0}\right)$ normalized to $\sum_{N} \Gamma_{N}^{\text {total }}=100 \%$.

\begin{tabular}{|c|c|c|c|c|c|c|c|}
\hline$m_{0}$ & 0 & 2 & 4 & 6 & 8 & 10 & $\Gamma_{N}^{\text {tota }}$ \\
\hline 2 & 2 & 1 & & & & & 3 \\
\hline 4 & 6 & 8 & 1 & & & & 15 \\
\hline 6 & 5 & 17 & 5 & 0.3 & & & 27 \\
\hline 8 & 2 & 15 & 9 & 1 & 0.03 & & 27 \\
\hline 10 & 0.7 & 7 & 8 & 2 & 0.1 & 0.003 & 18 \\
\hline & & & & & \multicolumn{3}{|c|}{$\sum_{N>12} \Gamma_{N}^{\text {total }}=10$} \\
\hline
\end{tabular}

directions, we take the fraction of all events with $K \bar{K}$ to be simply $2 \times(14 \%) \times 0.86=24 \%$. The actual fraction could be a bit larger. This includes decays $J \rightarrow 1 \gamma \rightarrow$ hadrons with a $K \bar{K}$ pair. The smallest identifiable branching ratio is for events with $N \bar{N}$. If we take twice the fraction containing $\bar{p}$ with momentum $p<1 \mathrm{GeV}$ we have $\approx 6 \%$ for this. We estimate the fraction $J \rightarrow N \pi$ ( $N$ even) to be $6 \%$, based on the modes $J \rightarrow 4 \pi^{c}, 6 \pi^{c}$ and the same statistical model as before, but now for $N$ even (keeping $\langle N\rangle=7$ ). In this way we can obtain

$$
\begin{aligned}
\Gamma(J \rightarrow \text { pions }) & +\Gamma(J \rightarrow \text { pions }+ \\
+ & \geqslant 1 K \bar{K}) \\
& =29 \% \text { pions }+N \bar{N}) \\
& 224 \%+6 \%=59 \%,
\end{aligned}
$$

excluding production of $\eta, \eta^{\prime}$ and also excluding possible radiative decays [e.g. $\left.J \rightarrow \gamma \eta^{\prime}, \gamma X(2.8)\right]$. The error in (18) is surely large $( \pm 10 \%$, say), and hard to estimate. The decays $\gamma \eta, \gamma \eta^{\prime}$ are negligible ${ }^{6,7}$ but $\gamma X(2.8)$ probably is not. The most likely candidates for most of the marginally "missing" $27 \%$ are decays of the type $J \rightarrow\left(\eta\right.$ or $\left.\eta^{\prime}\right)+$ pions $\rightarrow$ more pions, plus possible photons from $\eta$ or $\eta^{\prime}$ decays. We have already remarked that the fraction of events with $\eta+\eta^{\prime}$ could be as large as that with $K \bar{K}$. Notice that $G$-parity-conserving decays of
$J$ lead to final states (Npions) $+\left(\eta\right.$ or $\left.\eta^{\prime}\right)$ with $N$ odd. Thus, since $\eta$ or $\eta^{\prime}$ decays always contain $\geqslant 1$ neutral $\pi^{0}$ or $\gamma$, the final pion state in this case has always $\geqslant 2$ neutrals. On the other hand, the presence of $\eta$ and $\eta^{\prime}$ can only lead to final states with charged pions and one neutral through the $1_{\gamma}$ decay of $J$. Thils source of contamination is surely small, relative to $G$-conserving decays of $J$.

If it is possible to separate out the direct decay of $\psi^{\prime} \rightarrow(N-1) \pi^{c} 1 \pi^{0}$, sulotracting those that arise from $\psi^{\prime} \rightarrow J \pi \pi$, it should be possible to use these channels to guess the direct decay branching ratio for $\psi^{\prime} \rightarrow$ hadrons.

It is difficult to make any definite statements concerning the $C=+$ states at 3.4 and $3.5 \mathrm{GeV}$ at present. We only observe here that for the meson at $3.4 \mathrm{GeV}$, the branching ratios for $\psi^{\prime}$ into a photon plus $\pi^{+} \pi^{-}+K^{+} K^{-}, 4 \pi^{ \pm}$, or $6 \pi^{ \pm}$, quoted to be ${ }^{5}$ $(0.13 \pm 0.05) \%$, $(0.14 \pm 0.07) \%$, and $0.1 \%$, are consistent with Table III (assuming $\pi^{*} \pi^{-}=K^{+} K^{-}$). We can use this to guess at: a total pion branching ratio $\psi^{\prime} \rightarrow \gamma+\chi(3.4) \rightarrow \gamma+$ pions of $(2 \pm 1) \%$. Since kaon channels have been seen, we feel safe in multiplying this by $\approx 86 \% / 23 \%=3.7$ (the ratio of all hadrons to all pions at the $J$ ) so as to estimate a branching ratio $\psi^{\prime} \rightarrow \gamma+\chi(3.4)-\gamma+$ hadrons of $(7 \pm 3) \%$. The error is solely experimental. A similar number emerges for $P_{c}(3.5)$. Then the quoted branching ratio $\psi^{\prime} \rightarrow P_{c} \gamma \rightarrow J+\gamma \gamma=(4 \pm 2) \%$, and the fact that $\left(P_{c}^{\prime} \rightarrow J+\gamma\right) /\left(P_{c} \rightarrow J+\gamma\right)$ is roughly 2 events $/ 6$ events $=\frac{1}{3},{ }^{6}$ indicates that the hadronic decays of $P_{c}^{\prime}(3.4)$ are $70 \ldots 90 \%$ of the total $P_{c}^{\prime}(3.4)$ decay rate, if $P_{c}^{\prime}=\chi(3.4)$. For the $P_{c}(3.5)$, the estimated range is $50-70 \%$. Of course, this all assumes that only G-conserving hadronic or $J+\gamma$ decays occur.

Note added in proof. We wish to thank P. Jasselette for calling our attention to his extensive tables of Pais coefficients. ${ }^{18}$
${ }^{1} J$. J. Aubert et al ., Phys. Rev. Lett. 33, 1404 (1974). ${ }^{2} \mathrm{~J} .-\mathrm{E}$. Augustin et al., Phys. Rev. Lett. 33, 1406 (1974). ${ }^{3}$ G. S. Abrams et al., Phys. Rev. Lett. 33, 1453 (1974). ${ }^{4}$ DASP Collaboration, Phys. Lett. 57B, 407 (1975).

${ }^{5}$ G. J. Feldman et al ., Phys. Rev. Lett. 35, 821 (1975). ${ }^{6}$ B. Wiik, DESY Report No. DESY $75 / 37,1975$ (unpublished).

7J. Heintze, in Proceedings of the 1975 International Symposium on Lepton and Photon Interactions at High Energies, Stanford, California, edited by W. T. Kirk (SLAC, Stanford, 1976), p. 97.

${ }^{8}$ C. H. Llewellyn Smith and A. Pais, Phys. Rev. D $\underline{6}$, 2625 (1972).

${ }^{9}$ A. Pais, Ann. Phys. (N.Y.) 9 , 548 (1960).

${ }^{10}$ This method is more general; we only need it for $I=0$.

${ }^{11}$ For corresponding inequalities for prong branching ratios, see A. Pais, Phys. Rev. Lett. 32, 1081 (1974).

${ }^{12}$ We ignore the $J \rightarrow 1 \gamma \rightarrow I=1$ hadrons decay here.

${ }^{13}$ Many of the following have also been derived by F. Gilman, SLAC Report INo. SLAC-PUB-1600, 1975 (unpublished) .

${ }^{14} \mathrm{~S}$. Kitakado and T. F. Walssh, Lett. Nuovo Cimento 12 , 547 (1975).

${ }^{15}$ This has also been emphassized by H. Harari, Phys. Lett. $60 \mathrm{~B}, 172$ (1976). He infers a large $\eta^{\prime}$ fraction solely from the heavy-quark-antiquark content of the $\eta^{\prime}$.

${ }^{16}$ A. M. Boyarski et al ., SLAC Report No. SLAC-PUB1599/LBL-3897 (unpublished).

${ }^{17} \mathrm{C}$. Moorhouse, talk given at the SLAC Summer Symposium, 1975 (unpublished).

${ }^{18}$ P. Jasselette, Nucl. Phys. B23, 481 (1970). 